\title{
Susceptibility of Helicobacter pylori to Antibiotics Including Tigecycline
}

Rosa Monno ${ }^{1 *}$, Luciana Fumarola ${ }^{1}$, Carmen Capolongo ${ }^{1}$, Carla Calia ${ }^{1}$, Carlo Pazzani ${ }^{2}$, Enzo lerardi ${ }^{3}$ and Giuseppe Miragliotta ${ }^{4}$

${ }^{1}$ Department of Basic Medical Science, Neuroscience and Sense Organs, Policlinico Hospital, Bari, Italy

${ }^{2}$ Department of Biology, University of Bari, Bari, Italy

${ }^{3}$ Department of Emergency and Organ Transplantation, Policlinico Hospital, Bari, Italy

${ }^{4}$ Department of Interdisciplinary Medicine, Policlinico Hospital, Bari, Italy

\begin{abstract}
Background: $H$. pylori antibiotic resistance is an important factor in the treatment failure, therefore is important to know the local pattern of this resistance.

Material and Methods: A total of 111 patients were studied. Ninety- one $H$. pylori strains isolated from patients, including 12 from children, having previous repeated treatment failure and 20 strains were isolated from naïve patients, were studied. Antibiotic susceptibility including those to tigecycline, was determinated by E-Test.

Results: In treated adult and children patients the resistance rates were respectively $81 \%$ and $91.6 \%$ for clarithromycin; $27.8 \%$ and $41 \%$ for amoxicillin; $67.1 \%$ and $16.7 \%$ for metronidazole; $38 \%$ and $8.3 \%$ for levofloxacin $5.1 \%$ and $0 \%$ for tetracycline. Primary resistance, in naïve adult patients was $50 \%$ for clarithromycin, $10 \%$ for amoxicillin, $20 \%$ for metronidazole, $30 \%$ for levofloxacin and $0 \%$ for tetracycline. Tigecycline has shown good activity, in vitro, against $H$. pylori $\left(\mathrm{MIC}_{90}=0.064 \mathrm{mg} / \mathrm{L}\right)$.

Conclusion: The resistance rates found in $H$. pylori, in our area, are very high both in naïve and treated patients. Few papers have reported the tigecycline susceptibility in $\mathrm{H}$. pylori. The good activity and the lack of resistance to tigecycline found in our study, may consider this antibiotic a "rescue" therapy, saving the use of other antibiotics such as rifabutin, a drug used for the treatment of tuberculosis.
\end{abstract}

Keywords: H. pylori; Resistance; Tigecycline

\section{Introduction}

Helicobacter pylori is a Gram negative bacterium, well adapted to living in an unfriendly environment, the human stomach. An inflammatory reaction of the gastric mucosa detected by histology is present in only a few percent and few of these infected subjects present sufficient symptoms to seek medical attention. $H$. pylori gastritis may remain stable but, in $15-20 \%$ of the infected subjects, may progress to gastric and duodenal ulcers and atrophy of the gastric mucosa possibly resulting in adenocarcinoma and stomach lymphoma. The infecting $H$. pylori strains, the host response and environmental factors may all play a role in the different outcomes of the $H$. pylori infection [1]. Therefore the cure of this infection is mandatory in well-stated conditions [2]. A combination of a proton pump inhibitor with at least two antibacterial agents (clarithromycin, methronidazole, tetracycline, amoxicillin, levofloxacin) has been used to treat $H$. pylori infection. Resistance to these antibiotics has emerged and is one of the main factors in the failure of $H$. pylori eradication therapy. The prevalence of $H$. pylori antimicrobial resistance varies in different geographic areas, and it has been correlated with the consumption of antibiotics in the general population [3]. A recent systematic review of studies concerning primary $H$. pylori-antibiotic resistance in different continental areas has shown an overall resistance to the antibiotics commonly included in the first and second line therapy for $H$. pylori [4]. That is $11.2 \%$ for amoxicillin, $17.2 \%$ for clarithromycin, $26.7 \%$ for metronidazole, $5.9 \%$ for tetracycline and $16.2 \%$ for levofloxacin. Most interesting is the percentage of 1.4 of resistance to rifabutin, used as rescue therapy, and the $9.6 \%$ of reported multidrug resistance [5]. Tigecycline, the first member of the glicylcyclines, a new class of antibiotics structurally related to tetracyclines, is an antibiotic only recently commercially available. Tigecycline is very active against Gram-positive and Gramnegative, aerobe facultative bacteria and also against anaerobic bacteria [6]

In this study, we have evaluated the in vitro resistance of $H$. pylori to antibiotics used to treat the infection over a seven year period. The in vitro activity of tigecycline has also been assessed.

\section{Material and Methods}

\section{Patients}

Over a period of seven years (2006-2012), a total of 111 non repetitive $H$. pylori strains were isolated from gastric biopsies of 99 adult patients (age $\geq 18$ years) and 12 pediatric patients (age $<18$ years) suffering from dyspepsia and undergoing upper endoscopy at the Gastroenterology Unit of the University Hospital, Bari, Italy. The mean age of patients was 49.1 years and ratio M/F was 1:5. All patients consent the use of the data.

\section{Culture of H. pylori}

Culture was performed as previously reported [7,8]. Antral biopsies (and in 62 patients also body biopsies) were placed into saline solution plus $20 \%$ glucose and sent to the laboratory of Microbiology and Virology, University Hospital, Bari within $2 \mathrm{hrs}$. The smears from the biopsy specimens were stained by Gram stain. H. pylori was isolated on Pylori agar (Biomérieux, Florence, Italy). The isolates were identified by morphology at Gram stain and positivity for cytochrome oxidase, catalase, and urease tests.

*Corresponding author: Rosa Monno, Department of Basic Medical Science Neuroscience and Sense Organs, Policlinico Hospital, Bari, Italy, Tel: 0039 805448586; E-mail: rosa.monno@uniba.it

Received June 29, 2015; Accepted August 27, 2015; Published August 31, 2015

Citation: Monno R, Fumarola L, Capolongo C, Calia C, Pazzani C, et al. (2015) Susceptibility of Helicobacter pylori to Antibiotics Including Tigecycline. J Medical Microbiol Diagnosis S5:005. doi:10.4172/2161-0703.S5-005

Copyright: ( 92015 Monno R, et al. This is an open-access article distributed under the terms of the Creative Commons Attribution License, which permits unrestricted use, distribution, and reproduction in any medium, provided the original author and source are credited. 
Minimum Inhibitory Concentrations (MICs) were determined using the E-test methodology (AB Biodisk, Solna, Sweden) using Mueller Hinton agar medium plus $10 \%$ horse blood (Oxoid, Milan, Italy). The following antimicrobial agents were tested: amoxicillin, clarithromycin, levofloxacin, metronidazole, tetracycline, tigecycline. The MIC interpretative breakpoints used in this study have been those recommended by the European Committee on Antimicrobial Susceptibility Testing (EUCAST): clarithromycin: R $>0.5 \mathrm{mg} / \mathrm{L}$, amoxicillin $\mathrm{R}>0.12 \mathrm{mg} / \mathrm{L}$, metronidazole $\mathrm{R}>8 \mathrm{mg} / \mathrm{L}$, levofloxacin and tetracycline $\mathrm{R}>1 \mathrm{mg} / \mathrm{L}$. H. pylori ATCC 43504 was used as a control strain.

\section{Results and Discussion}

A total of 111 strains of $H$. pylori isolated from biopsy samples were tested. Ninety-nine $H$. pylori isolates were from adult patients (aged 1962 years, mean age 49.1 years, 37 women and $62 \mathrm{men}$ ). The other $12 \mathrm{H}$. pylori strains were isolated from children aged 5-9 years (mean age 6.2, 7 females and 5 males).

Twenty adult patients underwent endoscopy due to a variety of upper gastrointestinal symptoms and had never been pre-treated (naïve) for $H$. pylori infection. Ninety-one patients (79 adults plus 12 children) have a history of the failure of two or more therapeutic regimens (first line therapy: PPI plus amoxicillin plus clarithromycin for 10 days and in case of failure PPI plus metronidazole and clarithromycin for 10 days; if the infection is not eradicate, PPI plus amoxicillin plus levofloxacin is used). $\mathrm{MIC}_{50}$ and $\mathrm{MIC}_{90}$ of the $111 \mathrm{H}$. pylori isolates were $64 \mathrm{mg} / \mathrm{L}$ and $256 \mathrm{mg} / \mathrm{L}$ for clarithromycin; $0.032 \mathrm{mg} / \mathrm{L}$ and $96 \mathrm{mg} / \mathrm{L}$ for amoxicillin; $24 \mathrm{mg} / \mathrm{L}$ and $256 \mathrm{mg} / \mathrm{L}$ for metronidazole; $0.25 \mathrm{mg} / \mathrm{L}$ and $32 \mathrm{mg} / \mathrm{L}$ for levofloxacin; $0.094 \mathrm{mg} / \mathrm{L}$ and $0.5 \mathrm{mg} / \mathrm{L}$ for tetracycline; $0.032 \mathrm{mg} / \mathrm{L}$ and $0.064 \mathrm{mg} / \mathrm{L}$ for tigecycline, respectively.

The overall resistance rates were $26.1 \%$ for amoxicillin, $76.6 \%$ for clarithromycin, $33.3 \%$ for levofloxacin, $53.1 \%$ for metronidazole and $3.6 \%$ for tetracycline. In naive adult patients the resistance rate to amoxicillin was $10 \%$, to clarithromycin $50 \%$, to levofloxacin $30 \%$, to metronidazole $20 \%$ and $0 \%$ to tetracycline. In treated adult patients the H. pylori resistance rate was as follows: $27.8 \%$ to amoxicillin, $81 \%$ to clarithromycin, $38 \%$ to levofloxacin, $67.1 \%$ to metronidazole, $5.1 \%$ to tetracycline. In all treated pediatric patients, even if data refer to only twelve $H$. pylori strains, the resistance was $41.7 \%$ for amoxicillin, $91.7 \%$ for clarithromycin, $8.3 \%$ for levofloxacin, $16.7 \%$ for metronidazole and $0 \%$ for tetracycline (Table 1 ). All the strains studied resulted susceptible to tigecycline with very low $\mathrm{MIC}_{50}$ and $\mathrm{MIC}_{90}$ values $(0.032 \mathrm{mg} / \mathrm{L}$ and $0.064 \mathrm{mg} / \mathrm{L}$, respectively) and slightly lower when compared to corresponding values found for tetracycline.

Amoxicillin resistance rates found both in naïve and treated patients are very high compared to those found in other studies. We were unable

\begin{tabular}{|c|c|c|c|c|}
\hline \multirow[t]{2}{*}{ Antibiotics } & \multirow{2}{*}{$\begin{array}{l}\text { All Patients } \\
(\mathrm{N}=111) \\
\text { number }(\%)\end{array}$} & \multirow{2}{*}{$\begin{array}{c}\text { Naïve } \\
\text { Patients } \\
(\mathrm{N}=20) \\
\text { number (\%) }\end{array}$} & \multicolumn{2}{|c|}{$\begin{array}{c}\text { Treated Patients } \\
(\mathrm{N}=91) \\
\text { number (percentage) }\end{array}$} \\
\hline & & & $\begin{array}{c}\text { adults }(\mathrm{N}=79) \\
\text { number }(\%)\end{array}$ & $\begin{array}{c}\text { children }(\mathrm{N}=12) \\
\text { number }(\%)\end{array}$ \\
\hline Amoxicillin & $29(26.1)^{*}$ & $2(10)$ & $22(27.8)^{*}$ & $5(41.7)$ \\
\hline Clarithromycin & 85 (76.6) & $10(50)$ & $64(81)$ & $11(91.7)$ \\
\hline Levofloxacin & 37 (33.3) & $6(30)$ & $30(38.0)$ & $1(8.3)$ \\
\hline Metronidazole & $59(53.1)$ & $4(20.6)$ & $53(67.1)$ & $2(16.7)$ \\
\hline Tetracycline & $4(3.6)$ & $0(0)$ & $4(5.1)$ & $0(0)$ \\
\hline
\end{tabular}

* These percentages were corrected after that the resistance to amoxicillin was not confirmed on 14 out $32 \mathrm{H}$. pylori strains stored at $-80^{\circ} \mathrm{C}$ when re-evaluated with the agar dilution method (see text).

Table 1: Resistance rates in naïve adults and treated adults and children. to reconfirm amoxicillin resistance rates detected by E-test in 14 out 32 still viable strains (after storage at $-80^{\circ} \mathrm{C}$ ) isolated from treated adult patients re-evaluated with the agar dilution method. Similarly, Mégraud and coll. report that none of the 12 strains supposedly resistant to amoxicillin and also to tetracycline and rifabutin could be confirmed as such by agar dilution testing [3]. However the possibility that the amoxicillin resistance rates reported in this study is overestimated may not be ruled out.

Resistance to two antibiotics was found in $33.3 \%$ of the strains, in particular for clarithromycin plus metronidazole (11.7\%) followed by resistance to clarithromycin plus amoxicillin (9\%). Resistance to three antibiotics was detected in $26.1 \%$ of $H$. pylori strains, with the resistance to clarithromycin plus amoxicillin plus metronidazole found in $12.6 \%$ of strains, followed by resistance to clarithromycin plus metronidazole plus levofloxacin (10.8\%). Resistance to four and five antibiotics was found in $8.1 \%$ and $1.8 \%$ of $H$. pylori strains, respectively. Tigecycline has a potent in vitro activities against all $111 \mathrm{H}$. pylori tested and no increase in the MIC values were observed in the course of the years of the study and in particular following the introduction of tigecycline into clinical practice in Italy in 2007.

One of the factors responsible for the treatment failure of $H$. pylori infections is the increasing prevalence of $H$. pylori antibiotic resistance [4]. Primary clarithromycin resistance has emerged worldwide. In Italy, a primary resistance of $26.7 \%$ for clarithromycin and of $17.2 \%$ for metronidazole has been reported $[9,10]$. A study conducted to assess the primary antibacterial resistance rates of $H$. pylori in 18 European countries found a $H$. pylori resistance rate in adults of $17.5 \%$ for clarithromycin, $14.1 \%$ for levofloxacin, $34.9 \%$ for metronidazole, $0.7 \%$ for amoxicillin, $0.9 \%$ for tetracycline and $1.1 \%$ for rifabutin. However the resistance to rifabutin, amoxicillin and tetracycline was not confirmed when the MIC of these antibiotics was detected by agar dilution method. In the same study resistance rates for children were: $31.8 \%$ for clarithromycin, $25.7 \%$ for metronidazole, $2.5 \%$ for levofloxacin and $0.3 \%$ for amoxicillin. All the strains isolated from children were susceptible to tetracycline [3]. Earlier studies conducted in Italy showed that primary resistance to clarithromycin was high $(18.4 \%-26.7 \%)$ in Central and South Italy and similar to $23 \%$ reported in another Italian study [9-12]. These percentages are higher than $1.8 \%$ and $14 \%$ found in studies conducted about ten years ago $[13,14]$ suggesting an increase of resistance rate over time. In addition, these resistance rates may be higher due to the reported discordance between the two methods used to establish the resistance: E-Test and Real Time PCR, the latter detecting more resistant strains [7].

The prevalence of $H$. pylori antimicrobial resistance increases following previous ineffective treatment. A statistically significant difference has been found in the prevalence of antimicrobial resistance to metronidazole ( $27 \%$ vs $41.3 \%)$, clarithromycin (18\% vs $45.8 \%)$ and levofloxacin (3\% vs $14.6 \%$ ) in $H$. pylori-infected subjects who had never previously received an antibiotic treatment in comparison to patients who had failed one or more eradication treatments [15]. The percentages of resistance found in our treated adult patients are higher than those found by other Authors. The compliance of the patients, the number and the duration of the previous treatments and the local use of antibiotics in the empiric therapy of other infectious diseases, such as respiratory disease for which macrolides, fluorochinolones and amoxicillin are widely used in our area, may account for the differences in the data of the present study.

The high prevalence of antimicrobial resistance in patients naïve to treatment found in our study is probably due to the low number of 
patients tested. In our clinical practice, we have tested a major number of $H$. pylori strains isolated from patients with a history of failure of several previous therapeutic treatments. This is because we operate a work practice where culturing is limited to selected situations in which resistance is more likely to be encountered (failure of several lines therapies to eradicate the infection). In addition we have used the MIC interpretative breakpoint recommended by EUCAST and different in some cases, from those used by other Authors. Our levels of primary resistance to amoxicillin (10\% in adults) are higher than data reported by Mégraud and coll : $0.7 \%$, Pilotto and coll.: $1.8 \%$ and similar to those found by the De Francesco and coll.: 11\% and Savarino and coll. : 14\% $[3,4,13,14]$. A resistance (MIC> $256 \mathrm{mg} / \mathrm{L}$ ) of $31 \%$ to amoxicillin has been found in a study conducted on 43 patients in North Sardinia, Italy [16]. However it is very difficult to compare data on $H$. pylori resistance from various studies due to the different breakpoints used (EUCAST or arbitrarily chosen), the different bacterial inoculum used (2-4 McFarland standards), the different agar medium used, the percentage and type of blood added to the medium, the lack of a reference strains of $H$. pylori as control and last, but not least, the local use of antibiotics including amoxicillin that in our area is very diffuse in the form of amoxicillin/clavulanic acid. In addition in the majority of studies on prevalence of $H$. pylori antimicrobial resistance only the susceptibility to amoxicillin in $H$. pylori strains isolated from naïve patients is reported. The prevalence of $H$. pylori antimicrobial resistance increases following previous ineffective treatment [15]. All these factors may account for the differences observed. These differences may be due also to a mistake in the interpretation of the zone of inhibition of

E-test or to the loss of amoxicillin resistance after storage of strains at $-80^{\circ} \mathrm{C}$ as reported by other Authors. Stable and unstable resistance of H. pylori to amoxicillin has been reported [17]. This study, conducted on $7 \mathrm{H}$. pylori strains exhibiting high-level amoxicillin resistance (MIC $>256 \mathrm{mg} / \mathrm{L}$ ), shows that 3 of these strains lost their resistance after storage at $-70^{\circ} \mathrm{C}$ and reculture suggesting the existance of multiple mechanisms of resistance in $H$. pylori. Seventeen primary isolates of H. pylori strains (collected in Italy and USA) exhibiting amoxicillin resistance (and $\beta$-lactamase negative) lost amoxicillin resistance after storage of strains at $-80^{\circ} \mathrm{C}$. The resistance was rescued by plating these strains onto amoxicillin gradient plates. The ratios of MBC/MIC of amoxicillin ranged from $32 \mathrm{mg} / \mathrm{L}$ to $>1024 \mathrm{mg} / \mathrm{L}$ suggesting that these strains were tolerant to amoxicillin [18]. If this loss of amoxicillin resistance, after storage of $H$. pylori at $-80^{\circ} \mathrm{C}$, also occurred in our case, remains to be established.

To explain these discrepancies requires further investigations, as also the understanding the precise reasons why the reading of the inhibition zones of antibiotics such as tetracycline, metronidazole, levofloxacin, clarithromycin and tigecycline is easy, whereas amoxicillin is difficult to read due to a lack of a clear zone of inhibition.

Several clinical trials and meta-analyses have shown that the first-line therapies (proton pump inhibitors PPIs plus amoxicillin and clarithromycin or PPIs plus amoxicillin and metronidazole) may fail in about $20 \%$ of patients. Therefore several "rescue" therapies have been proposed and used. Tigecycline is the first member of the glycylcyclines, a new class of broad-spectrum antibacterials that are structurally related to tetracyclines that were developed to overcome the two major mechanisms of tetracycline resistance (ribosomal protection and efflux) [19].

Tigecycline was originally approved by the Food and Drug Administration (FDA) and the European Medicines Agency (EMA) for the treatment of complicated intra-abdominal and skin and soft tissue infections and more recently also for the treatment of other infections $[20,21]$. The drug is taken up into human polymorphonuclear neutrophils with an intracellular concentration 20-30 times higher than extracellular concentrations Recent studies have also shown that tigecycline is effective in inhibiting bacteria in biofilms. In the light of recent evidence suggesting that $H$. pylori forms biofilm either in vitro or in vivo on the gastric mucosa epithelium new insight has been gained in the therapeutic strategies to adopt against $H$. pylori [22]. The failure of several antibiotic therapy lines, even if, in vitro, the bacterium result susceptible to all antibiotics, may be due to the growth of $H$. pylori on the gastric mucosa as "biofilm persister cells", tolerant to antibiotics and the subsequent failure of antibiotics to penetrate the biofilm matrix.

Few data are reported in the literature on the in vitro activity of tigecycline against $H$. pylori strains. Tigecycline has potent in vitro activities against $H$. pylori with $\mathrm{MIC}_{50}$ and $\mathrm{MIC}_{90}$ values of $0.03 \mu \mathrm{g} / \mathrm{ml}$ and $0.06 \mu \mathrm{g} / \mathrm{ml}$, respectively (range $0.015-0.12 \mu \mathrm{g} / \mathrm{ml}$ ). These values were fourfold lower than those of tetracycline $\left(\mathrm{MIC}_{90}=0.25 \mu \mathrm{g} / \mathrm{ml}\right)$ also found in our study [23].

Khosravi and coll., have tested various antibiotics against $H$. pylori including tigecycline but no data are reported in the Abstract available [24].

The excellent in vitro activity of tigecycline in $H$. pylori isolates will be potentially very useful in rescue therapy for the $H$. pylori infections although there are disadvantages such as tigecycline is not recommended for pregnant women or children younger than 8 years due to the inhibition of bone growth and the dental staining, the need for intravenous administration and the cost of therapy of about 84-85 US \$ per day.

Tigecycline has shown promising in vitro activity against $H$. pylori. These interesting results obtained in vitro should be strengthened by in vivo studies considering that, even with the current most effective treatment regimens, the eradication of $H$. pylori infection will not succeed in about $20 \%$ of patients. Tigecycline treatment can be a "rescue therapy" in all patients with a history of treatment failure and in particular in those infected by strains of $H$. pylori harboring the cagA gene which are associated with the most severe manifestation of $H$. pylori infection. The use of tigecycline will also save the use of the rifabutin, the antibiotic used for the treatment of infections due to Multidrug-resistant Mycobaterium tuberculosis and diseases caused by infection with other Mycobacterium spp.

Knowing the resistance rates also in patients with repeated treatment failure can open a window on the transmission and increased diffusion of resistant $H$. pylori strains. This knowledge can be very useful locally for the choice of also new antibiotics to be used in the empiric therapy of $H$. pylori infection.

\section{Competing Interests}

The authors declare that they have no competing interest exists.

\section{References}

1. Montecucco C, Rappuoli R (2001) Living dangerously: how Helicobacter pylori survives in the human stomach. Nat Rev Mol Cell Biol 2: 457-466.

2. Malfertheiner P, Mégraud F, O'Morain CA, Atherton J, Axon AT, et al. (2012) Management of Helicobacter pylori infection-the Maastricht IV/Florence Consensus Report. Gut 61: 646-664.

3. Mégraud, F, Coenen SA, Versporten M, Kist M, Lopez-Brea M, et al. (2013) Helicobacter pylori resistance to antibiotics in Europe and its relationship to antibiotic consumption. Gut 62: 34-42.

4. De Francesco V, Giorgio F, Hassan C, Manes G, Vannella L, et al. (2010) Worldwide H. pylori antibiotic resistance: a systematic review. J Gastrointestin 
Citation: Monno R, Fumarola L, Capolongo C, Calia C, Pazzani C, et al. (2015) Susceptibility of Helicobacter pylori to Antibiotics Including Tigecycline. J Medical Microbiol Diagnosis S5:005. doi:10.4172/2161-0703.S5-005

Liver Dis 19: 409-414.

5. Glocker E, Bogdan C, Kist M (2007) Characterization of rifampicin-resistant clinical Helicobacter pylori isolates from Germany. J Antimicrob Chemother 59: 874-879.

6. Frampton JE, Curran MP (2005) Tigecycline. Drugs 65: 2623-35.

7. Monno R, Giorgio F, Carmine P, Soleo L, Cinquepalmi V, et al. (2012) Helicobacter pylori clarithromycin resistance detected by Etest and TaqMan real-time polymerase chain reaction: a comparative study. APMIS 120: 712-717.

8. Mégraud F, Lehours $P(2007)$ Helicobacter pylori detection and antimicrobial susceptibility testing. Clin Microbiol Rev 20: 280-322.

9. De Francesco V, Margiotta M, Zullo A, Hassan C, Valle ND, et al. (2006) Primary clarithromycin resistance in Italy assessed on Helicobacter pylori DNA sequences by TaqMan real-time polymerase chain reaction. Aliment Pharmaco Ther 23: 429-435.

10. De Francesco V, Margiotta M, Zullo A, Hassan C, Valle ND, et al. (2006) Clarithromycin resistance and Helicobacter pylori genotypes in Italy. J Microbiol 44: $660-664$

11. De Francesco V, Zullo A, lerardi E, Giorgio F, Perna F, et al. (2010) Phenotypic and genotypic Helicobacter pylori clarithromycin resistance and therapeutic outcome: benefits and limits. J Antimicrob Chemother 65: 327-332.

12. Toracchio S, Marzio L (2003) Primary and secondary antibiotic resistance of Helicobacter pylori strains isolated in central Italy during the years 1998-2002. Dig Liver Dis 35: 541-545.

13. Pilotto A, Rassu M, Leandro G, Franceschi GM, Di Mario F (2000) Prevalence of Helicobacter pylori resistance to antibiotics in North-east Italy: a multicentre study. Interdisciplinary Group for the Study of Ulcer. GISU. Dig Liver Dis 32: 763-768.

14. Savarino V, Zentilin P, Pivari M, Bisso G, Mele MR, et al. (2000) The impact of antibiotic resistance on the efficacy of three 7-day regimens against Helicobacter pylori. Aliment Pharmacol Ther 14: 893-900.
15. Romano M, lovene MR, Russo MI, Rocco A, Salerno R, et al. (2008) Failure of first-line eradication treatment significantly increases prevalence of antimicrobial-resistant Helicobacter pylori clinical isolates. J Clin Pathol 61: $1112-1115$

16. Dore MP, Piana A, Carta M, Atzei A, Are BM, et al. (1998) Amoxicillin resistance is one reason for failure of amoxicillin-omeprazole treatment of Helicobacter pylori infection. Aliment Pharmacol Ther 12: 635-639.

17. Han SR, Bhakdi S, Maeurer MJ, Schneider T, Gehring S (1999) Stable and unstable amoxicillin resistance in Helicobacter pylori: should antibiotic resistance testing be performed prior to eradication therapy? J Clin Microbio 37: $2740-2741$.

18. Dore MP, Osato MS, Realdi G, Mura I, Graham DY, et al. (1999) Amoxicillin tolerance in Helicobacter pylori. J Antimicrob Chemother 43: 47-54.

19. Greer ND (2006) Tigecycline (Tygacil): the first in the glycylcycline class of antibiotics. Proc (Bayl Univ Med Cent) 19: 155-161.

20. Tanaseanu C, Milutinovic S, Calistru PI, Strausz J, Zolubas M, et al. (2009) Efficacy and safety of tigecycline versus levofloxacin for community-acquired pneumonia. BMC Pulm Med 9: 44.

21. Bates D, Parkins M, Hellweg R, Gibson K, Bugar JM (2012) Tigecycline treatment of urinary tract infection and prostatitis: case report and literature review. Can J Hosp Pharm 65: 209-215.

22. Cammarota G, Sanguinetti M, Gallo A, Posteraro B (2012) Review article: biofilm formation by Helicobacter pylori as a target for eradication of resistant infection. Aliment Pharmacol Ther 36: 222-230.

23. Yang JC, Lee PI, Hsueh PR (2010) In vitro activity of nemonoxacin, tigecycline, and other antimicrobial agents against Helicobacter pylori isolates in Taiwan, 1998-2007. Eur J Clin Microbiol Infect Dis 29: 1369-1375.

24. Khosravi Y, Teh XS, Ramli NSK, Gunaletchumy SP, Loke MF, et al. (2012) Helicobacter pylori virulence and antibiotic susceptibility pattern in Malaysian patients. Proceedings of the $15^{\text {th }} I C I D$, Bangkok.
This article was originally published in a special issue, Molecular Biology \& Genetics of Pathogens handled by Editor(s). Dr. Daniel Olusola Ojurongbe, University of Tuebingen, Germany 